\title{
What Makes Us Smell: The Biochemistry of Body Odour and the Design of New Deodorant Ingredients
}

\author{
Andreas Natsch* \\ KGF-SCS Industrial Investigator Award 2014
}

\begin{abstract}
Today, axilla odours are socially stigmatized and are targeted with deodorants and antiperspirants representing a multi-billion market. Axilla odours aren't simple byproducts of our metabolism but specifically formed by an intricate interplay between i) specific glands, ii) secreted amino acid conjugates of highly specific odorants and iii) selective enzymes present in microorganisms colonizing our skin, providing a natural 'controlledrelease' mechanism. Within a multidisciplinary research project, we were able to elucidate the structure of key body odorants, isolate and characterize secreted amino acid conjugates and identify the enzymes responsible for odour release. These enzymes then served as targets for the development of specific active compounds in an almost medicinal chemistry approach, an approach rarely used in the cosmetic field so far. Here we review the key new insights into the biochemistry of human body odour formation, with some remarks on the experimental steps undertaken and hurdles encountered. The development of deodorant actives and the difficult path to market for such specifically acting cosmetic actives is discussed. The basic insights into the biochemistry also opened the way to address some questions in population genetics: Why have large proportions of Asians lost the 'ability' to form body odours? Do twins smell the same? Are our typical body odours indeed influenced by the immune system as often claimed? After addressing these questions, I'll conclude with the key remaining challenges in this field on an ecological niche that is 'anatomically very close to our heart'.
\end{abstract}

Keywords: Aminoacylase · Controlled release · Deodorants · Human body odours

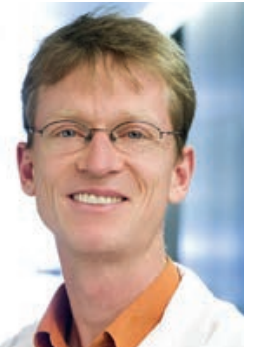

Andreas Natsch studied at the Swiss Federal Institute of Technology and got his $P h D$ in environmental microbiology at the Institute for Plant Sciences of the ETHZ. After short postdoctoral studies in molecular biology at the Centro Nacional de Biotecnologia in Madrid, he moved to the research department of the fragrance manufacturer Givaudan. Here he focused on

${ }^{\star}$ Correspondence: Dr. A. Natsch

Givaudan Schweiz AG

Ueberlandstrasse 138

$\mathrm{CH}-8600$ Duebendorf, Switzerland

Tel.: +41448242105

E-mail: andreas.natsch@givaudan.com the elucidation of the biochemical mechanisms of body odour formation in order to design new deodorant ingredients. In parallel he investigated the biological activities of fragrance raw materials and developed novel formulations, e.g. for preservation of cosmetic products. Since 2008, the research focus has gradually shifted to the development and implementation of in vitro assays to study toxicological risks associated with cosmetic and fragrance ingredients without using animal testing. This work focused first on the mechanism and the kinetics of the reactivity of skin allergens with peptides and then led to the development of the KeratinoSens ${ }^{\mathrm{TM}}$ assay to study allergens. This assay was globally adopted in 2015 by the OECD as the first biological animal-free assay to screen for skin allergens.

\section{The Importance of Skin Bacteria in Odour Formation and the Origin of Species}

The human axilla is covered by a dense array of secretory glands. Apocrine channels associated with hair follicles secrete a hydrophobic mixture containing fats, steroids and proteins while eccrine glands pro- vide water and hydrophilic contents such as salts and lactic acid. These secretions serve as ideal growth medium for a number of bacterial species. At the same time the anatomy of the axilla and the dense coverage by hair provide a moist environment - making the axilla an almost perfect habitat for bacterial colonisation leading to a dense bacterial population, which was already described decades ago. ${ }^{[1]}$

A direct association between the skin bacteria and body odour formation was recognized at an early stage: Fresh apocrine secretions are odourless, but they develop the typical pungent odour if contacted with skin bacteria. ${ }^{[2]}$ These initial observations prepared the ground to search for the mechanism of body odour formation, which could only be understood by focusing on both the skin microflora and the axilla secretions to elucidate the interplay between both contributors. Surprisingly, in the fifty years following the recognition of the importance of skin colonizing bacteria for odour formation, no enzyme in these organisms was described, which would explain the bacteria-triggered odour release. This left us a largely unexplored field!

Two main bacterial genera colonize the axilla: Staphylococci and Corynebacteria, and odour formation could already in early 
studies be associated with the population density of Corynebacteria ${ }^{[1,3]}$ and only these bacteria were able to release odour when incubated with axilla secretions. Thus it would have appeared straightforward where to start the search for the relevant enzymes and biochemical transformations. However, when we entered this field, we realized that the public global depositories for bacterial isolates, which had collected thousands of bacterial strains including specimens from all sorts of exotic niches such as Antarctic soils or hydrothermal vents, did not cover a single isolate of Corynebacteria from the human axilla. Hence, at the start of our studies we took advantage of a 6-day igloo expedition (see Fig. 1) with friends to the Swiss Mountains, where, in absence of western-world hygienic amenities, the natural balance of the axilla flora could re-establish before it was sampled prior to a much needed subsequent visit to a thermal spa. Some strains isolated at this event (in particular strain Ax20 referred to below) were identified as Corynebacteria in agreement with literature findings and served in all biochemical steps of the project - but first chemical analysis was required in order to identify the odorants and the chemical substrates leading to odour formation.

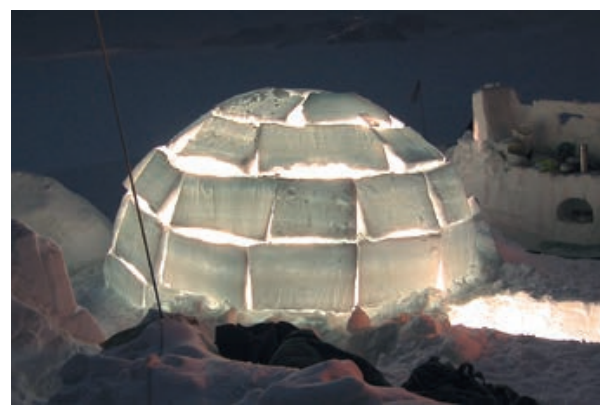

Fig. 1. After spending six days in an igloo camp in the Alps, the 'true wilderness' had re-established on the skin surface of the author's friends and representative, odour-forming Corynebacteria were isolated prior to a spa visit.

\section{Specific Odorants and Odorant Precursors Secreted in the Human Axilla}

\subsection{Volatiles}

The chemistry of odorants in the axilla had been studied before we entered the field: Two odoriferous steroids, originally described as key odorants and pheromones in the pig, $5 \alpha$-androst-16-en-3-on and $5 \alpha$-androst-16-en-3 $\alpha$-ol (Scheme 1), were the first odorants identified, although analytically detectable levels are extremely low in human sweat. While these steroids do smell at low concentrations, there is an unusually high frequency of anosmia, with up to $50 \%$ of the human population having an odour-blindness for $5 \alpha$-androst-16-en3-on.

The odorant acid 3-methyl-2-hexenoic acid $(3 \mathrm{M} 2 \mathrm{H})^{[4]}$ was the second principle identified. It was originally found in schizophrenic patients (and thus first coined 'schizophrenic acid') but later detected in individuals in the general population, too. We found that this is not the dominant odorant acid in sweat, but that the closely related 3-hydroxy-3-methyl-hexanoic acid (HMHA) is even more abundant and quantitatively the most dominant human odorant. ${ }^{[5]}$ It has a very low detection threshold and our nose is able to detect levels as low as $0.0044 \mathrm{ng} / \mathrm{L}$ of air. With both a carboxylic acid and a hydroxyl group strongly reducing volatility and enhancing water solubility, this is an odorant with very unusual physicochemical properties. These properties probably also explain why it had escaped analytical detection for a long time, despite the fact that up to millimolar concentrations are sometimes present in sweat: In classical gas chromatographic analysis, it has an extremely low response factor (mainly due to quenching in injection ports and broad peak shapes reducing sensitivity).

Later we found that these two carboxylic acids are only the two quantitatively most prominent examples of a very diverse class of branched or unbranched, saturated, unsaturated or hydroxylated acids. ${ }^{[6]}$
The third class of odorants, which we found and published in parallel with two other research groups, is present in axilla secretions in minute quantities only: Different sulfanylalkanols with carbon skeletons similar to the previously identified acids. The common carbon skeletons point to a common biochemical origin, but the biosynthetic route to the key odorants is currently unknown. In Scheme 1 the structure of the key odorants known today are summarized.

\subsection{Precursors for the Odorants: Amino Acid Conjugates}

Early studies have shown that incubating secretions from apocrine glands with bacteria leads to odour formation. Therefore it was clear that either the odorants are simple metabolites directly formed by bacterial metabolism or that a 'controlled release' mechanism is at play, with the bacteria performing only the last step and releasing the odorants from preformed and secreted precursors.

Simple metabolism does explain the generation of foot or oral malodour. In these cases odour is formed by catabolic reactions of commensal microorganisms working on simple essential amino acids such as Met, Cys, Leu, Lys, Ile and Trp leading to very common volatile metabolites (e.g. sulfides, short branched acids, amines, indole and skatole).

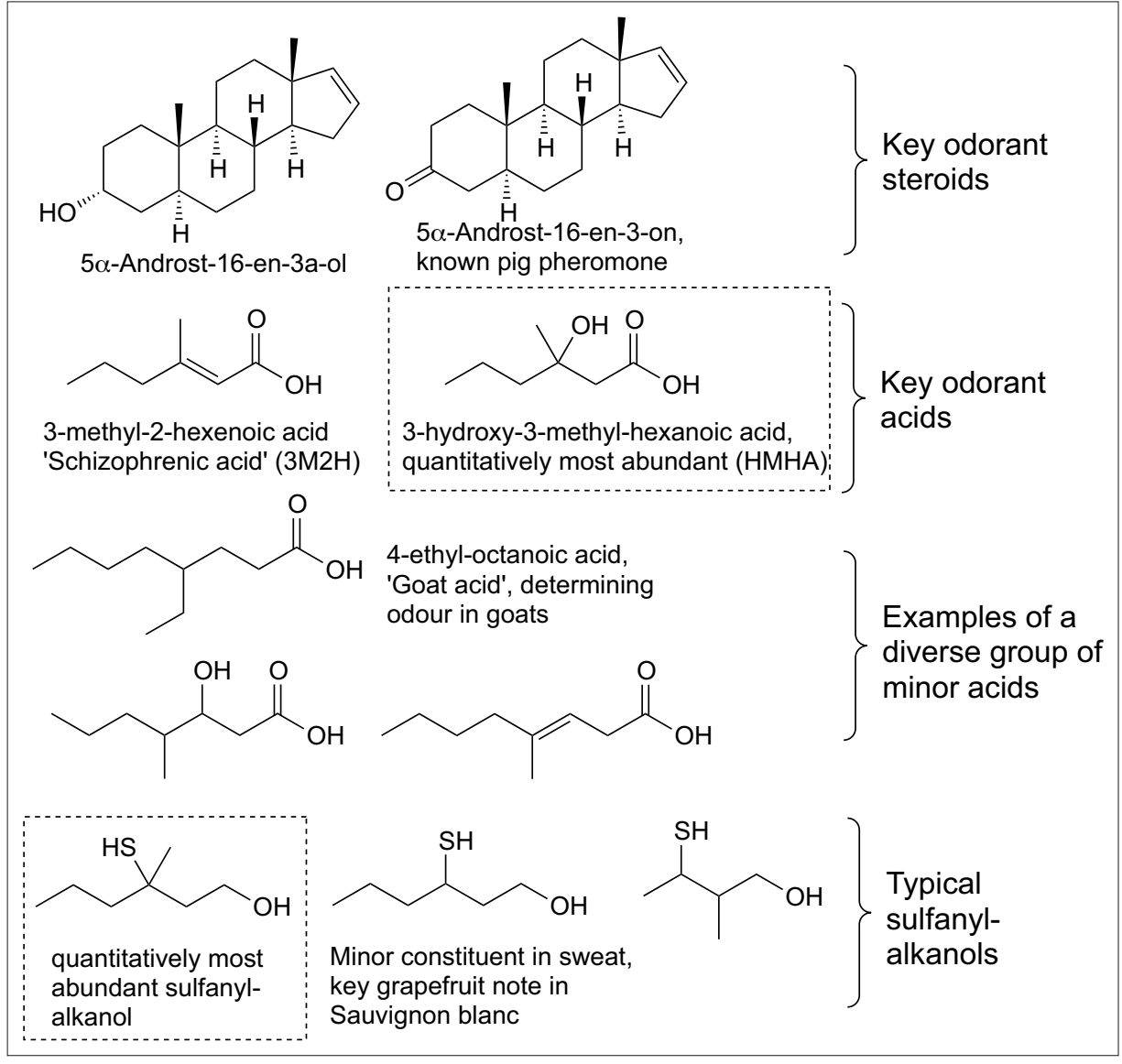

Scheme 1. The known key human axilla odorants. 
Given the very specific structure of the odorants described above, we and others suspected that the cleavage of preformed precursors is at play in the case of axilla odour. Thus in a pioneering study on the release of $3 \mathrm{M} 2 \mathrm{H}$, it was shown that this compound is bound to a water-soluble precursor and can be liberated therefrom both by hydrolysis catalyzed by $\mathrm{NaOH}$ or by bacterial action using corynebacterial isolates. [7] However these studies could not identify the structure of the precursors.

Using a combination of multiple methods (size exclusion chromatography, LCMS, hydrolysis of sweat fractions and sensorial analysis) we could show that axilla secretions contain large amounts of glutamine conjugates, in which the acids $3 \mathrm{M} 2 \mathrm{H}$ and HMHA are covalently attached to the $\mathrm{N}_{\alpha}$-atom of glutamine as shown in Scheme 2. ${ }^{[5]}$ This observation was later confirmed by an independent laboratory quantifying the HMHA-Gln conjugate over time on 49 volunteers. ${ }^{[8]}$ Based on all available evidence, glutamine conjugates appear to be the key precursors for odorant acids.

Originally we proposed that the sulfanylalkanols are secreted as cysteine conjugates based on indirect evidence, namely that i) Corynebacterium strain Ax20 can release the odorants from such synthetic cysteine conjugates, and that ii) a cystathionine- $\beta$-lyase cloned from this bacterial strain can release the odorants from both the synthetic cysteine conjugates and from authentic sweat (see below). ${ }^{[9]}$ However this early study did not identify the suspected precursors directly in axilla secretions.

It was later shown that the quantitatively dominating precursor actually is a cysteine-glycine conjugate (Scheme 2). [10] This finding was confirmed by our laboratory ${ }^{[11]}$ and in later population studies. ${ }^{[8,12]}$ A minor level of the originally proposed cysteine conjugate was then also analytically detected,[11] although it is not clear whether this compound is directly secreted by the glands or whether it is an intermediate formed on the skin by a peptidase activity.

\section{Malodour-releasing Enzymes in Axilla Bacteria}

Although the importance of bacteria and the contribution of specific taxonomic groups to odour formation had long been known, the quest to identify odour-releasing enzymes could only start once the precursors/substrates were analytically identified and synthetic samples of these compounds were available. Using these substrates, we could identify particular bacterial strains in the collection of bac-

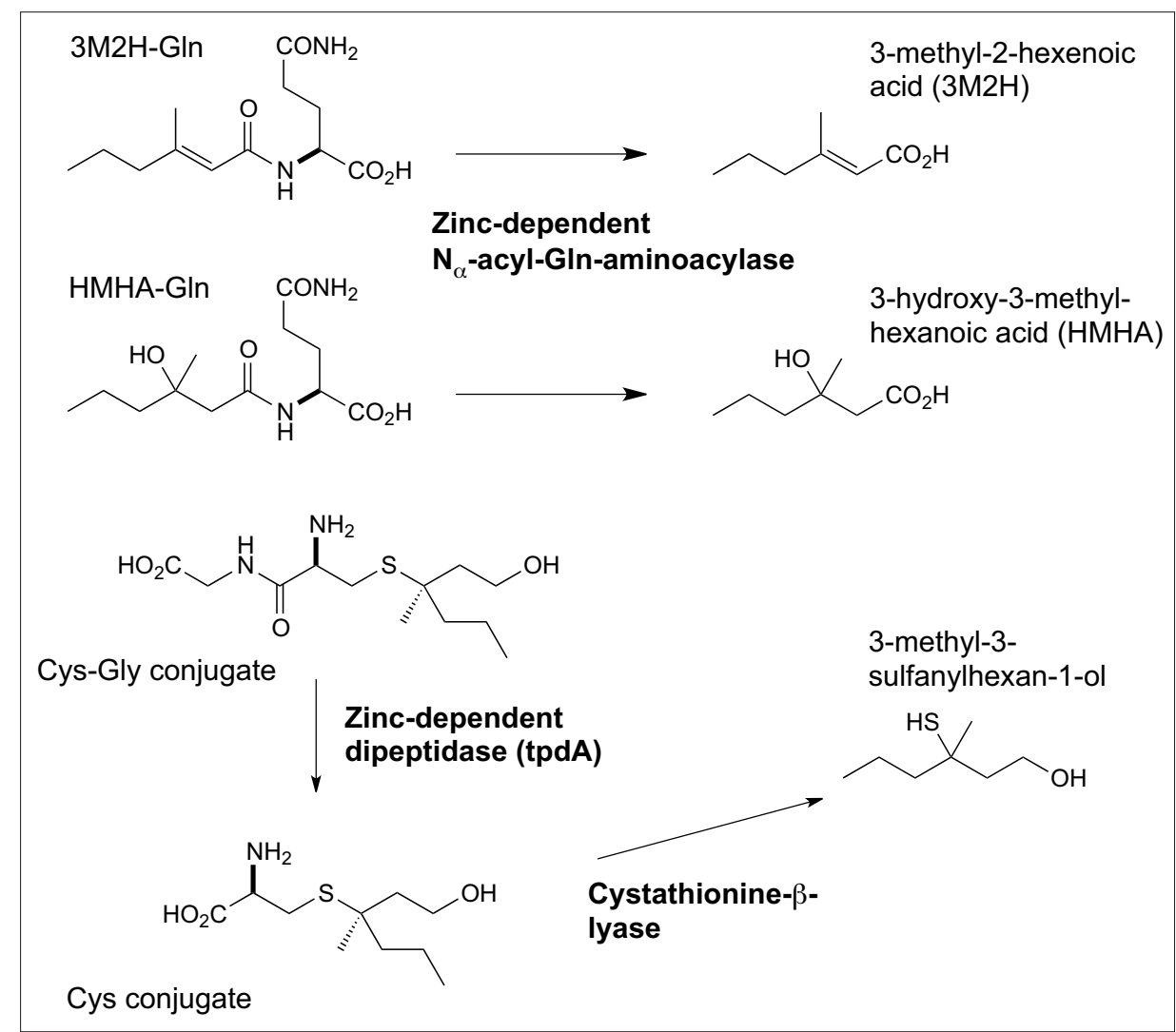

Scheme 2. The structure of the secreted amino acid conjugates and the three enzymatic activities in Corynebacterium Ax20 responsible for odour release.

teria sampled at the end of the igloo trip with the specific ability to cleave the amino acid conjugates. A highly odour-forming strain, Corynebacterium sp. Ax20,[5] was selected as model organism for subsequent biochemical work.

\subsection{The Acid-releasing Enzyme}

The first focus was to explain the release of the most dominant odorants, the carboxylic acids. Ax20 contained a unique intracellular enzyme activity releasing the acids from Gln-conjugates, and this could be pinpointed to an enzyme migrating as a single activity peak in all column separations subsequently performed. Using classical biochemical tools, this enzyme was thus purified to homogeneity and then used for amino acid sequence analysis. The corresponding gene was cloned and expressed in $E$. coli. ${ }^{[5]}$ The recombinant enzyme, biochemically speaking it is a $\mathrm{N}_{\alpha}$-acylglutamine aminoacylase, did cleave both the synthetic precursors and it released the acids from axilla secretions, ${ }^{[6]}$ thus verifying its role in odour release (Scheme 2). This enzyme was coined AMRE ( $\underline{\text { Axillary }}$ Malodour Releasing Enzyme). ${ }^{[13]}$ It has a high specificity for the glutamine residue in the substrate, not accepting other amino acids. At the same time it has a high tolerance to different acyl-chains linked to glutamine. We could later show that there are many different acyl-glutamine conjugates present in the human axilla, but no conjugates with other amino acids were found so far. Thus the substrate specificity of this bacterial enzyme (high specificity for Gln, but not the acyl part of the substrate) is perfectly adapted to the available substrates provided by the human host, and presents a fascinating example of coevolution of the skin bacteria with its host. The recombinant enzyme could be produced in high quantities, it was crystallized in complex with a specific inhibitor and the three-dimensional structure was solved. It is a homodimer with two active sites and it contains two zinc-atoms at each active centre. The structure is closely related to other bacterial zinc-dependent metallopeptidases. Using the recombinant enzyme, a high-throughput screening assay was designed, allowing the rapid screening for inhibitors (see below).

\subsection{The Enzymes Releasing \\ Sulfanylalkanols}

Based on the assumption that sulfanylalkanols might be linked to cysteine in the secreted form, we first synthesized cysteine conjugates in absence of analytical evidence for their presence in axilla secretions. These substrates are indeed cleaved by the same bacterial isolate Ax20. Thioethers of cysteine are substrates for cystathionine- $\beta$-lyases, which are encoded in many bacteria by the metC gene. The corresponding enzyme could therefore be cloned from Ax 20 by a genetic approach 
instead of the tedious classical biochemical approach of enzyme purification: Chromosomal fragments of Ax20 complementing $E$. coli met $C$ mutants were isolated. These fragments contained a common open reading frame, which was expressed in $E$. coli and the recombinant protein was purified. Also for this enzyme a rapid fluorescent assay to screen inhibitors was developed.

This enzyme indeed cleaved synthetic cysteine conjugates and it was able to release sulfanylalkanols from sweat (see Scheme 2), indicating that this pathway is relevant and that corresponding substrates exist in sweat. [9] A phylogenetically related cystathionine- $\beta$-lyase, which is also able to release sulfanylalkanols from cysteine conjugates, was later cloned from Corynebacterium jeikeium K411, [14] showing that this enzymatic activity is present in several species of Corynebacteria.

Upon the report that the key precursor for sulfanylalkanols is a Cys-Gly conjugate rather than a simple cysteine conjugate (see above), the question arose whether this compound could directly be cleaved by the $\beta$-lyase, but we found this not to be the case. On the other hand, enzyme extracts of Ax20 were able to release sulfanylalkanols from Cys-Gly conjugates, but this ability was lost as soon as the extracts were fractionated by column chromatography, pointing to the involvement of at least two enzymes. Indeed, if the recombinant $\beta$-lyase was added to individual chromatographic fractions, only then a fraction was identified which was able to release the odorant from the Cys-Gly conjugate. This indicated that a second enzyme is needed to first release the Gly-residue. This conclusion proved correct and led to the isolation, by classical purification and activity assays, of a novel dipeptidase named $t p d A$, which cleaved the glycine from the CysGly conjugate. This enzyme then generates the substrate for the previously identified $\beta$-lyase and the sequential action of both enzymes is needed for odour release. ${ }^{[11]}$ These findings are also summarized in Scheme 2.

\section{Towards More Specific Deodorants}

Over the last century, deodorants have mainly used three principles: i) Blocking bacterial growth by applying antibacterial actives (e.g. Triclosan), ii) using odour masking fragrances to cover the odours and iii) reducing the perspiration by using different inorganic salts, mainly aluminium chlorohydrate, which is thought to act by clogging sweat ducts. The specific nature of the biochemical reactions leading to odour formation now opens up the pos- sibility for a more targeted intervention by directly blocking the relevant biochemical reactions.

\subsection{Carbamates}

We designed different classes of potential active ingredients, using the new in vitro screening tests and the understanding of the substrate specificity. We first synthesized Gln-carbamates (Scheme 3) based on the knowledge that only $\mathrm{N}_{\alpha}$-substituted glutamines are accepted as substrates by the aminoacylase, and that a large range of different hydrophobic $\mathrm{N}_{\alpha}$-substituents is tolerated. These agents have a dual activity: If present in excess over the natural substrate, they reduce malodour release by competition because they are recognized as alternative substrates. ${ }^{[13]}$ In addition, they do release the fragrant alcohols selected for the synthesis, providing some enzymatic triggered fragrance release. However, the degradation by the enzyme comes with the disadvantage that an excess of the active compound over the natural substrate is needed. These agents therefore must be supplied in the millimolar range.

\subsection{Phosphinic Acid-derived Inhibitors}

Based on the knowledge that AMRE is a zinc-dependent metallopeptidase, active functional groups used in medicinal chemistry for the design of metallopeptidase inhibitors were applied to the basic acyl-Gln structure of the substrate. A first genera-

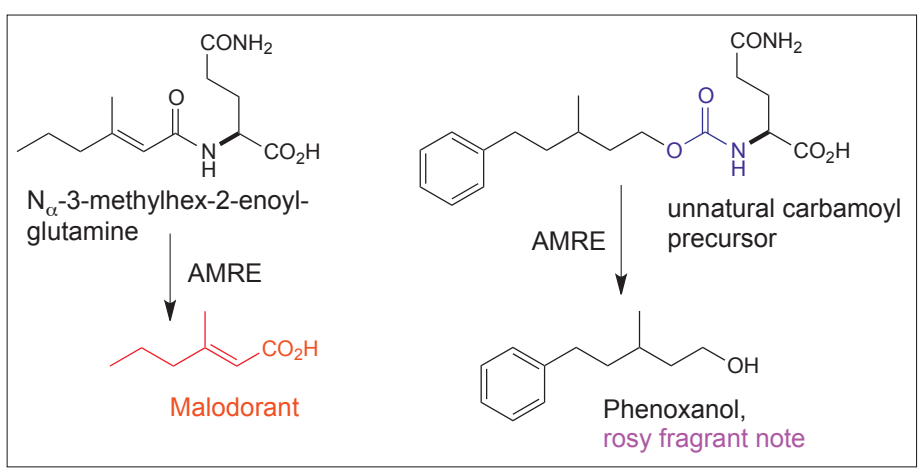

Scheme 3.

Carbamates acting as alternative substrates were designed as first generation of potential deodorant ingredients.

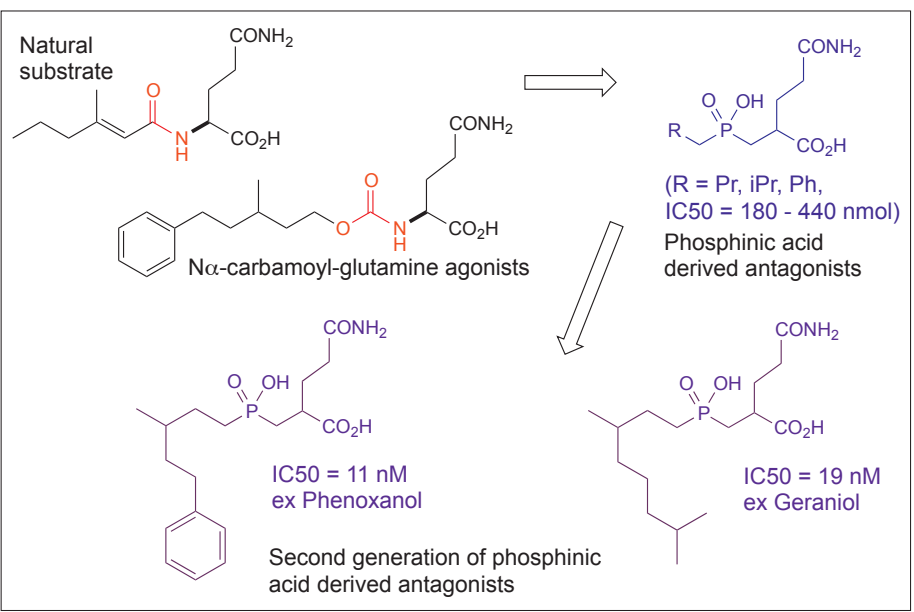

Scheme 4. The design of derivatives of phosphinic acid as highly active AMRE inhibitors. 


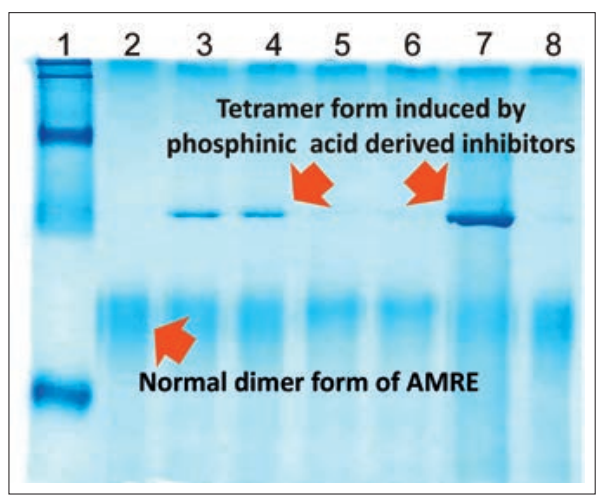

Fig. 2. The recombinant aminoacylase (lane 2 and 8) was preincubated with three inhibitors derived from phosphinic acid (lane 3,4,7) and two normal competitive inhibitors (lane 5 and 6). A stable tetrameric form surviving gel electrophoresis in a native gel was formed by preincubation with the phosphinic inhibitors only.

tors have the highly unusual ability to trigger a change in the enzymes' quaternary structure upon binding, leading to a very stable complex. We suggest this explains the kinetic behaviour similar to irreversible inhibitors.

\subsection{In vivo Activity: Carbamates vs. Phosphinic Acid-derived Inhibitors}

The most active carbamate and the best phosphinic acid-derived inhibitor were then taken into a series of in vivo tests. They were formulated into deodorant formulations without classical deodorant principles and applied to the axilla of panellists. Odour formation was evaluated by expert evaluators. The carbamate did reduce malodour formation in vivo, and this effect was most pronounced on individuals with high malodour scores (Fig. 3 ). These were also the panellists having high $\mathrm{N}_{\alpha}$-acyl-glutamine aminoacylase activity as determined by a noticeable release of the fragrant principle phenoxanol from the alternative substrate ${ }^{[16]}$ (Fig. 3). However, to our surprise, the phosphinic acid-derived inhibitors exhibited no in vivo activity when tested on the same panel of test subjects and using the same methodology despite the high in vitro activity. This lack of activity was confirmed in a second independent study. It is currently unclear whether this is due to poor absorption of the inhibitors by the bacteria, or maybe due to the very unusual mode of action (catalyzing tetramer formation in vitro) of this class of inhibitors discussed above.

\subsection{Urea Derivatives and Market Perspective}

The lesson learned from the above in vivo testing is that active ingredients most closely related to an actual substrate will probably provide best in vivo activity. This may be due to efficient uptake into the bacteria of close substrate-mimics. Thus the
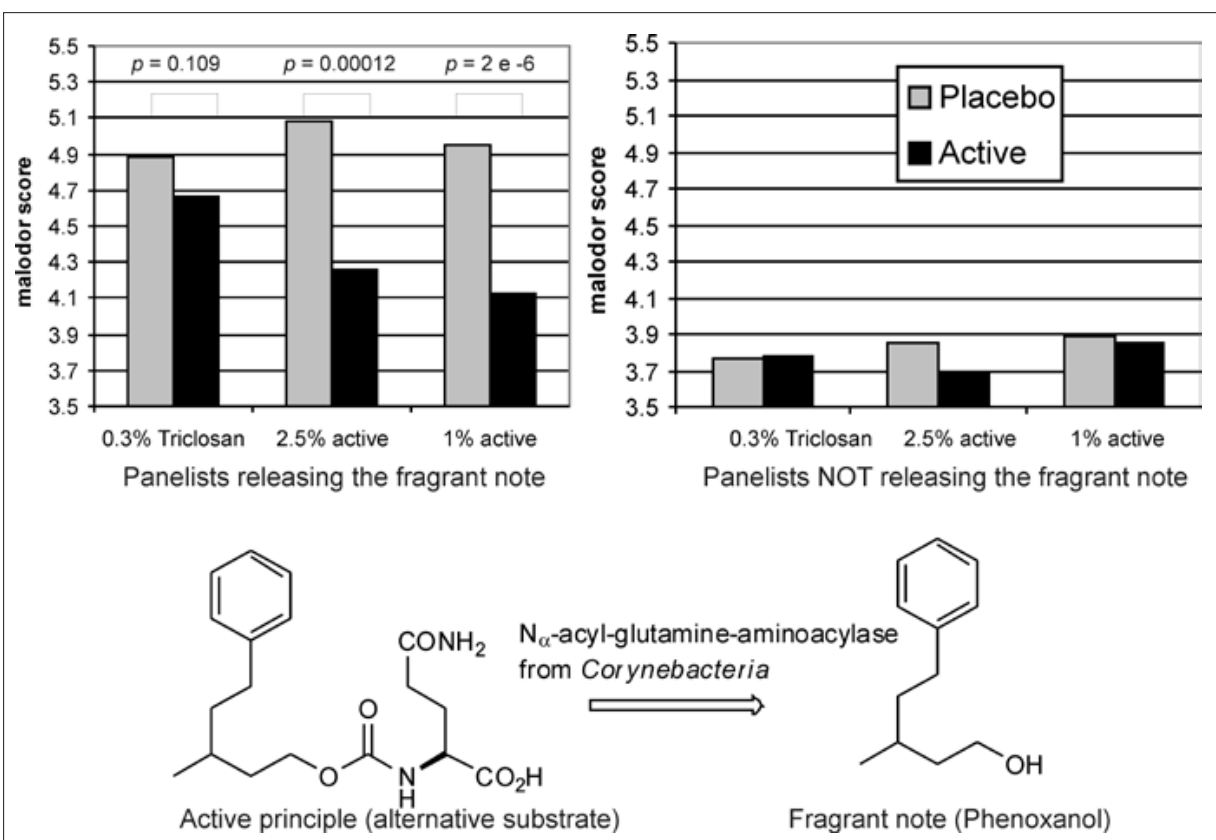

Fig. 3. In vivo test of an alternative substrate targeting the $\mathrm{N}_{\alpha}$-acyl-glutamine aminoacylase. Shown are malodour scores $8 \mathrm{~h}$ after application of deodorants containing either the classical principle Triclosan or the new active carbamate. Panelist results were separated for those on whom the fragrant note could be perceived by the assessors and those who did not release the fragrant note. The odour level was low in the 'negative panelists' putatively not carrying a bacterial population with high aminoacylase activity. At the same time no further malodour reduction could be observed in these panellists (right), but a clear effect was noted on individuals with high odour and carrying a bacterial flora able to cleave the substrate (left). Adapted from ref. [16]. last series of inhibitors which we developed are simple urea derivatives, closely related to the carbamates (Scheme 5). ${ }^{[17]}$ These compounds apparently bind to the active site but are not cleaved by the enzyme and thus provide more stable inhibition at lower concentration. At the same time, probably due to the close relationship with the natural substrate, they are active on different bacterial strains (Table 1). This latter fact is highly relevant as we found in the meantime that there is a high biodiversity in the human axilla with different bacterial strains all belonging to Corynebacteria but having highly divergent gene sequences.

At $10 \mathrm{ppm}(0.033 \mathrm{mM})$ an average inhibition of $80 \%$ is achieved when testing the best candidate of this series on living bacteria, i.e. in an experiment taking uptake by the bacteria into account. Promising in vivo results were found for this ingredient and the fact that it so closely mimics a natural substrate (Scheme 5) present on the skin may also lead to an acceptable safety profile.

However, due to the very cheap availability and high efficacy of aluminium salts and also due to the good odour coverage provided by carefully designed perfumes, the specifically acting ingredients discussed above have not yet made their way to market, partly due to cost considerations. This may change in the near future, because currently there is an increased

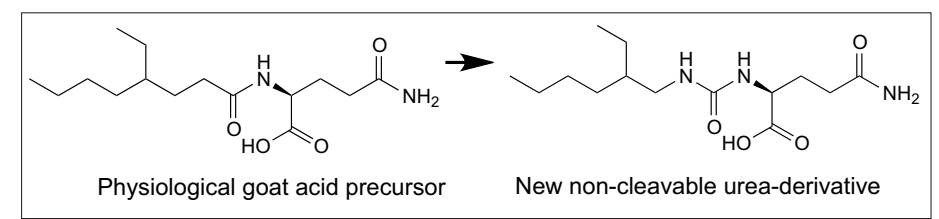

Table 1. In vitro activity of the urea-derivative on five genetically distinct Corynebacteria. Data

Conc. [ppm] Inhibition of release of 3-hydroxy-3-methyl-hexanoic acid [\%]

\begin{tabular}{|l|l|l|l|l|l|l|} 
& Ax20 & Ax30 & Ax43 & Ax52 & Ax73 & Av. \\
\hline 10 & 51 & 95 & 93 & 77 & 107 & 84.6 \\
\hline 100 & 81 & 100 & 97 & 83 & 87 & 89.6
\end{tabular}
from ref. [17].
Scheme 5. The design of a non-cleavable urea derivative closely mimicking a natural substrate. 
pressure on the use of aluminium salts in personal care products triggered by some alleged toxicological risks. Even if these purported risks may not hold up to a rigorous scientific assessment, mass media coverage and internet gossip have spread these concerns and the pressure on industry to provide aluminium-free products is rising. Two recent reports from public authorities have highlighted uncertainty in the toxicological exposure assessment to aluminium, further promoting consumer concerns (European SCCS opinion on the safety of aluminium in cosmetic products and German Bfr opinion No. 007/2014 on aluminium in antiperspirants). These developments may affect how specifically acting deodorant ingredients and the higher costs of products containing them are viewed in the future.

\section{The ABCC11 Polymorphism - An 'Evolutionary Knockout'}

A significant fraction of the population in the Far East is known to produce dry and white earwax, as opposed to the yellow and wet earwax dominant in the remaining global population. Interestingly, individuals with the 'dry and white' phenotype were reported to not have typical axilla odours. ${ }^{[18,19]}$ This phenotype was linked to the mutation of a single nucleotide to the 'A-allele' in the gene coding for the efflux pump protein ABCC11.[20] This mutation leads to a loss of function of this transport protein (Fig. 4). Thus, if the release and subsequent cleavage of amino acid conjugates described above is the key mechanism of odour formation, then one would infer that the carriers of the inactive form of the ABCC11 efflux pump should have lost the ability to secrete the amino acid conjugates.

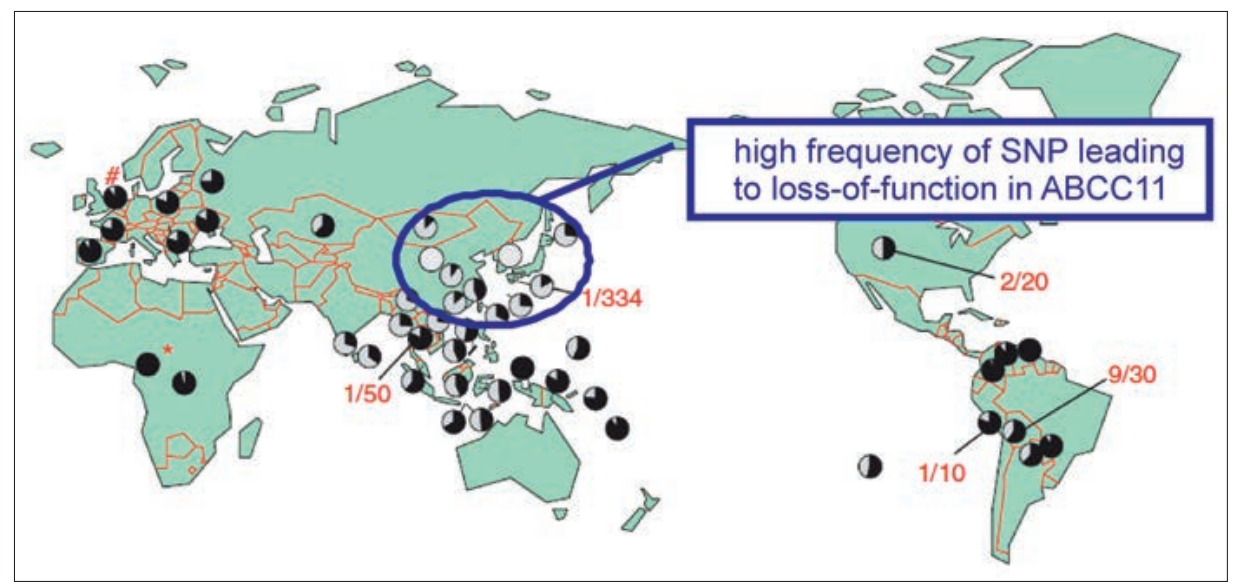

Fig. 4. Global frequency of the 'A-allele' in the ABCC11 gene in the human population. Grey indicates frequency of the mutated 'A-allele'. Individuals carrying this loss-of-function mutation on both chromosomes have lost completely the ability to secrete the amino acid conjugates listed in Scheme 2, whereas these compounds are detectable in all individuals carrying one or two copies of the functional 'G-allele' on their chromosomes. Adapted from ref. [20]. dant of the mice MHC), ${ }^{[22]}$ although subsequent studies showed the findings to be controversial.[23]

Could thus a secreted odour-fingerprint indeed confer genetic information to a potential partner, and more specifically information on one's HLA-type? To address this question we first performed a twin study, and could show that indeed identical twins do have almost identical patterns of odorant acids released from Gln conjugates. Twins not only look the same, they smell the same! Hence, there is clearly a genetically conferred odour-fingerprint in human individuals. ${ }^{[24]}$

In a second step, the same study was extended to families with four siblings of identical sex, as only within families there is a high likelihood to find pairs with identical HLA-genotype. Again the pattern of odorant carboxylic acids was investigated and the individuals were typed for their HLA locus. However, we could not find a significant association between identical HLA-genotype and more closely related odour-fingerprints. ${ }^{[25]}$ Given the negative results in this study, the question remains open, whether new, genetically and HLAdetermined odorants can be isolated from human body secretions, or whether potential HLA-associated body odours in humans remain a myth as long as their chemical nature cannot be resolved by analytical techniques.

A new twist to this controversial field comes from a very recent report. Based on the earlier finding in mice, that specific synthetic peptides can trigger a response in mouse olfactory neurons, ${ }^{[26]}$ it was hypothesized that also humans may perceive nonapeptides, which are known as HLA-ligands. Such synthetic peptides were tested both in a behavioural study and in a neuroimaging study. Statistically significantly different results were obtained depending on the receiver's HLAgenotype, which led to the conclusion that such HLA-binding nonapeptides indeed are the HLA-dependent body odorants in human body secretions. [27] However, the study left many questions unanswered, as neither was there analytical verification of the HLA-dependent presence of such peptides in sweat nor evidence for conscious detection by human panellists - all parameters firmly established for the human body odorants described above. Finally, the question how non-volatile nonapeptides may reach the human olfactory epithelium remained unanswered.[28] Thus, until the mystery of the 'scent of love' is resolved, the dictum 'further research is needed' may hold for the years to come in this controversial field.

Another open question refers to the 'scent of fear' - several behavioural and neurological studies have shown that hu- 
mans can recognize the specific body odours emitted by persons under acute stress or in fearful situations, ${ }^{[29,30]}$ and this odour may confer a warning signal. Although this research subject lingers around in the neuroscience and behavioural literature for over a decade, no analytical studies pinpointing this odour to specific chemicals has been published so far. This odour appears to be very rapidly formed, which is logical if it was to serve as warning signal to conspecifics. Thus direct secretion of the odorants by the glands, probably triggered by alarm hormones, and no involvement of slow, bacteria-mediated controlled release appears likely. So far we could not identify any chemical involved in this typical odour, which we found difficult to sample. However it could be a highly rewarding field to study this odour at the chemical level for those interested in chemical ecology: Such a scent of fear may be a chemical heritage we still carry from our times in caves and igloos as a specific signal to warn our horde: Beware, there are bears and ice bears out there!

\section{Acknowledgement}

The studies reviewed here were highly collaborative and interdisciplinary, and I had the privilege to coordinate them. I'd like to thank all those involved: Chemical synthesis: F. Schroeder, F. Flachsmann, S. Derrer, T. Granier; Analytics: J. Schmid, F. Kuhn, H. Gfeller and G. Acuna. Biochemistry: R. Emter, M. Wasescha, W. Stauch, T. Haupt; Protein crystallography: A. Douangamath, J. Baker (EvoTec); ABCC11 study: Beiersdorf
Hamburg. For providing sweat samples, axilla bacteria and DNA probes thanks to all the volunteers. For all discussions and support my special thanks go to my supervisors Georg Fráter, Markus Gautschi and Boris Schilling.

Received: May 27, 2015

[1] J. J. Leyden, K. J. McGinley, E. Holzle, $J$. Invest. Dermatol. 1981, 77, 413.

[2] W. B. Shelley, H. J. Hurley, A. C. Nichols, Arch Derm. Syphilol. 1953, 68, 430.

[3] N. Shehadeh, A.M. Kligman, J. Invest. Dermatol. 1963, 41, 39.

[4] X. N. Zeng, J. J. Leyden, H. J. Lawley, K. Sawano, I. Nohara, G. Preti, J. Chem. Ecol. 1991, 17, 1469

[5] A. Natsch, H. Gfeller, P. Gygax, J. Schmid, G. Acuna, J. Biol. Chem. 2003, 278, 5718.

[6] A. Natsch, S. Derrer, F. Flachsmann, J. Schmid, Chemistry \& Biodiversity 2006, 3,1 .

[7] X. N. Zeng, J. J. Leyden, J. G. Brand, A. I. Spielman, K. J. McGinley, G. Preti, J. Chem Ecol. 1992, 18, 1039.

[8] M. Troccaz, G. Borchard, C. Vuilleumier, S. Raviot-Derrien, Y. Niclass, S. Beccucci, C. Starkenmann, Chem. Senses 2009, 34, 203.

[9] A. Natsch, J. Schmid, F. Flachsmann, Chemistry \& Biodiversity 2004, 1, 1058.

[10] C. Starkenmann, Y. Niclass, M. Troccaz, A. J. Clark, Chemistry \& Biodiversity 2005, 2, 705.

[11] R. Emter, A. Natsch, J. Biol. Chem. 2008, 283, 20645.

[12] A. Martin, M. Saathoff, F. Kuhn, H. Max, L. Terstegen, A. Natsch, J. Invest. Dermatol. 2010, 130, 529.

[13] A. Natsch, H. Gfeller, P. Gygax, J. Schmid, Int. J. Cosmetic Sci. 2005, 27, 115 .

[14] A. G. James, C. J. Austin, D. S. Cox, D. Taylor, R. Calvert, FEMS Microbiol. Ecol. 2013, 83, 527.

[15] F. Schroeder, M. Fournie-Zaluski, A. Natsch, Chem. Abstr. 2004, 141, 38737.
[16] A. Natsch, C. Joubert, M. Cella, F. Flachsmann, C. Geffroy, Flavour Fragrance J. 2013, 28, 262.

[17] A. Natsch, T. Granier, Int. Pat. Appl. WO2012056014 (A1), 2012.

[18] Y. Toyoda, A. Sakurai, Y. Mitani, M. Nakashima, K. Yoshiura, H. Nakagawa, Y. Sakai, I. Ota, A. Lezhava, Y. Hayashizaki, N. Niikawa, T. Ishikawa, FASEB J. 2009, 23, 2001.

[19] M. Nakano, N. Miwa, A. Hirano, K. Yoshiura, N. Niikawa, BMC Genet. 2009, 10, 42.

[20] K. Yoshiura, A. Kinoshita, T. Ishida, A. Ninokata, T. Ishikawa, T. Kaname, M. Bannai, K. Tokunaga, S. Sonoda, R. Komaki, M. Ihara, V. A. Saenko, G. K. Alipov, I. Sekine, K. Komatsu, H. Takahashi, M. Nakashima, N. Sosonkina, C. K. Mapendano, M. Ghadami, M. Nomura, D. S. Liang, N. Miwa, D. K. Kim, A. Garidkhuu, N. Natsume, T. Ohta, H. Tomita, A. Kaneko, M. Kikuchi, G. Russomando, K. Hirayama, M. Ishibashi, A. Takahashi, N. Saitou, J. C. Murray, S. Saito, Y. Nakamura, N. A. Niikawa, Nat. Genet. 2006, 38, 324.

[21] K. Yamazaki, G. K. Beauchamp, M. Curran, J. Bard, E. A. Boyse, Proc. Natl. Acad. Sci. USA 2000, 97,10500

[22] C. Wedekind, T. Seebeck, F. Bettens, A. J. Paepke, Proc. R. Soc. B 1995, 260, 245.

[23] S. C. Roberts, L. M. Gosling, V. Carter, M. Petrie, Proc. R. Soc. B 2008, 275, 2715.

[24] F. Kuhn, A. Natsch, J. R. Soc. Interface 2009, 6, 377.

[25] A. Natsch, F. Kuhn, J. M. Tiercy, J. Chem. Ecol. 2010, 36, 837.

[26] M. Spehr, K. R. Kelliher, X. H. Li, T. Boehm, T. Leinders-Zufall, F. Zufall, J. Neurosci. 2006 26, 1961.

[27] M. Milinski, I. Croy, T. Hummel, T. Boehm, Proc. R. Soc. B. 2013, 280, 20122889

[28] A. Natsch, Proc. R. Soc B 2014, 281, 20131678

[29] L. R. Mujica-Parodi, H. H. Strey, B. Frederick, R. Savoy, D. Cox, Y. Botanov, D.Tolkunov, D. Rubin, J. Weber, PLOS ONE 2009, 4, e6415.

[30] K. Ackerl, M. Atzmueller, K. Grammer, Neuro. Endocrinol. Lett. 2002, 23, 79. 